\title{
Efficient organic NLO material: Charge density analysis and device fabrication
}

Kunal Kumar Jha ${ }^{1}$, Shashi Bhushan Srivastava ${ }^{2}$, Samarendra Pratap Singh ${ }^{2}$, Parthapratim Munshi ${ }^{1}$ ${ }^{1}$ Department Of Chemistry Shiv Nadar University, Dadri, India, ${ }^{2}$ Department of Physics, Shiv Nadar University, Dadri, India E-mail: kk703@snu.edu.in

Organic scaffolds such as nitro stilbene having $n-\mathrm{e}^{-}$donor (D) acceptor (A) substituents facilitates inter/intra-molecular charge transfer, leading to the generation of good frequency conversion materials [1]. The interactions such as $\mathrm{C}-\mathrm{H} \bullet \bullet \bullet \mathrm{O}, \mathrm{C}$ $-\mathrm{H} \bullet \bullet n, C-H \bullet \bullet N$ etc. present in such D-ח-A system act as a channel for intermolecular charge transfer. Further, the presence of non-centric crystal field facilitates the charge transfer and hence the dipolar interactions; leads to the enhancement of dipole moment. Such D-ח-A systems in crystalline form having dense molecular packing serve as a good candidate for high mobility charge transport diode and field effect transistor [2]. In this direction, here we report the case of (Z)-3-(4-(dimethylamino)phenyl)-2-(4-nitrophenyl)acrylonitrile (NNDM_CNS), a cyano-substituted nitro stilbene derivative belongs to the family of D-n-A chromophore and crystallized in non-centrosymmetric space group, Pn. Detailed charge density analysis [3] based on both high resolution ( $\sin \theta / \lambda=1.1 \AA^{-1}$ ) X-ray diffraction data and theoretical structure factors derived via periodic calculations followed by topological analysis of intermolecular interactions and examination of electrostatic potential maps helped understanding the charge transfer mechanism in this system. Further, the intramolecular charge transfer states were experimentally observed in its emission spectra. Nonlinear optical (NLO) activity measurement and in-crystal NLO property calculations confirmed that this organic material is indeed a potential NLO material. Furthermore, the material was characterized for its charge transport properties and applications in organic electronic devices. The space charge limited current (SCLC) measurements were carried out using Ag/NNDM_CNS-single crystal/Ag and Cu/NNDM_CNSsingle crystal/Cu based electron-only and hole-only diodes, respectively. Additionally, Cu/NNDM_CNS-single crystal/Ag diode was fabricated and tested to study its bipolar charge transport characteristics. The combination of experimental and theoretical analyses along with complementary solution based and in-crystal studies suggest that this organic NLO material indeed an efficient charge transport material and it found to exhibit appropriate properties.

[1] Vrcelj, R.M. et al. (2002), Cryst. Growth Des. 2, 609-617.

[2] Liu, J. et al. (2015), Nat. Comm. 6, 10032.

[3] Hansen, N. K. and Coppens, P. (1978) Acta Cryst. A34, 909-921.
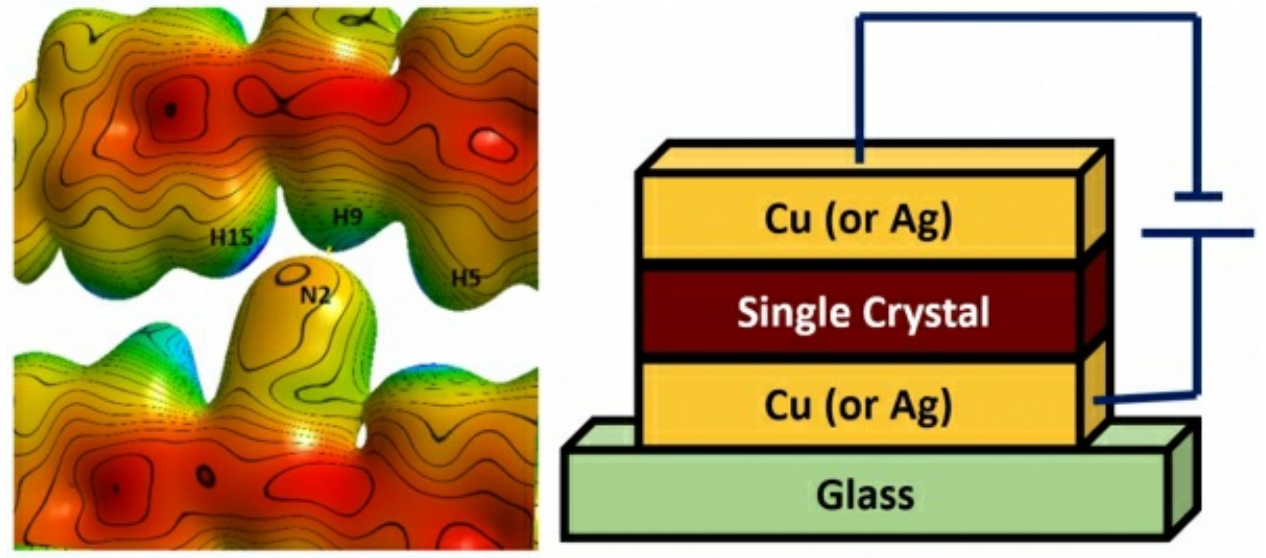

Charge density based electrostatic potential map (left) and schematic of diode prototype device (right).

Keywords: charge transfer material, charge density analysis, NLO material. 